\title{
4
}

\section{Evaluating Force Main Transients with SWMM5 and Other Programs}

\section{Karen E. Ridgway}

The question of whether SWMM5 and other hydraulic calculation programs commonly used to model wastewater collection systems can accurately simulate the flow conditions in pump station force mains was raised and discussed in the SWMM Users Group in 2008. Rises in pressure occur in the downstream force main following a pump turning on, and drops in pressure occur following pumps turning off. These changes are water hammer and result in pressure waves and flow rate oscillations in the force main.

Four one-dimensional hydraulic calculation programs were compared in the analysis of a theoretical force main problem. All of the programs are link and node type programs. The links may be channels, conduits, valves, gates, dams, weirs or pumps (depending on the program). The links are connected to junctions at either end. The junctions may be reservoirs, manholes, or closed conduit junctions such as pipe tees.

The programs evaluated include: SWMM5 by the United States Environmental Protection Agency, Water Hammer and Mass Oscillation (WHAMO) by the United States Army Corps of Engineers, the Transient Analysis Program (TAP) by Applied Science, Inc., and InfoWorks CS (Collection System) by Wallingford Software Ltd.

SWMM5 has a new force main feature that was tested. This feature did not exist in earlier versions of the SWMM program. Conduits may be specified as cylindrically shaped force mains that always flow full and have friction losses calculated using the Hazen-Williams or Darcy-Weisbach formulae.

Ridgway, K. 2010. "Evaluating Force Main Transients with SWMM5 and Other Programs." Journal of Water Management Modeling R236-04. doi: 10.14796/JWMM.R236-04.

(C) CHI 2010 www.chijournal.org ISSN: 2292-6062 (Formerly in Dynamic Modeling of Urban Water Systems. ISBN: 978-0-9808853-3-0) 


\subsection{Vented or Not Vented Junctions}

In SWMM5 and InfoWorks CS, the model junctions are always assumed to be vented. The drop in pressure in the force main following a pump turning off is limited to the invert elevation of the connecting network link. The hydraulic grade line (HGL) elevation that is calculated may not fall below the invert elevation of the connecting link. Air at atmospheric pressure is assumed to enter the network being modeled if the HGL falls below the connecting link crown elevation.

In WHAMO, the closed conduit calculations are always made in the network links regardless of the HGL being calculated or the network link elevations.

In TAP, junctions can be specified as either vented or sealed (not vented) with closed conduit or open channel calculations made in the connecting links depending on the user specified junction type, the calculated HGL, and the connecting link elevations.

Vented junctions work in the same fashion in TAP as with SWMM5 and InfoWorks CS. Sealed junctions in TAP work in the same fashion as junctions in WHAMO. With sealed junctions, the connecting conduits or gates are assumed to be full unless the conduit is vented from another direction. The HGL elevations can fall below the link crown elevation with less than atmospheric (vacuum) conditions calculated in the adjacent conduit cells.

\subsection{Air Entrance Valves}

In reality, force mains may be vented by installing air and vacuum valves on high points of the force main. These valves release trapped pockets of air when the pressure rises and allow air to enter the force main when large pressure drops occur. The entrance of air prevents the pressure from dropping below atmospheric pressure in the force main preventing vapor cavities from forming and collapsing (i.e. cavitation).

The capacity and location of the air and vacuum valves in pump station discharge headers/force mains is commonly evaluated using software programs such as WHAMO. This is especially true for large capacity pump stations with long force mains with the potential for significant cavitation damage.

The issue raised in the SWMM Users Group is not related to the design of air and vacuum valve systems at pump stations and in force mains, but whether SWMM5 and other programs used to model wastewater collection systems can 
reasonably simulate the flow rate in the downstream force mains as pumps turn on and off.

\subsection{Force Main Problem}

A typical force main problem was designed to have a pump station that lifts flow from a low reservoir into a force main that runs into a high reservoir. A plan and profile view of the force main problem is shown on Figure 4.1.

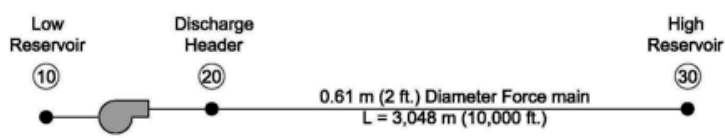

Plan View

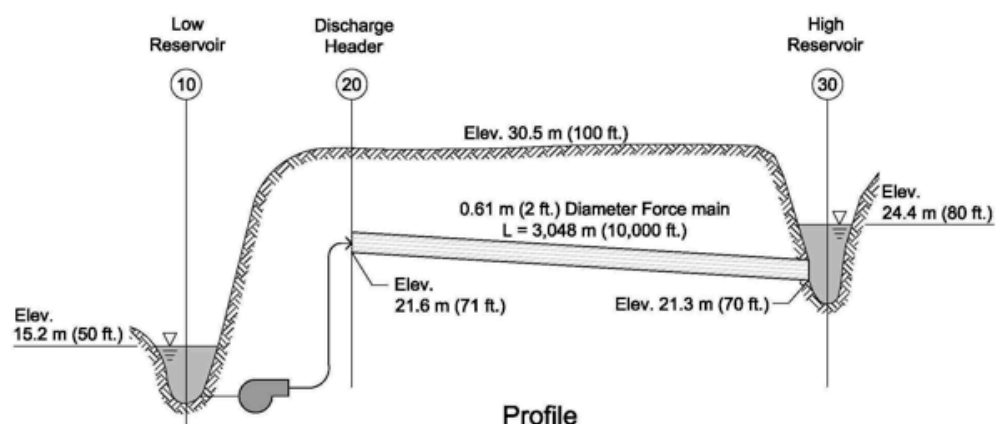

Figure 4.1 Plan view and profile of the force main problem.

This problem was kept simple to allow the various programs to simulate the geometry. In setting up the models, close attention was paid to whether the elements in each model were being represented similarly by all four programs.

Initially, the pump was assumed to be off and the HGL elevation in the force main at rest at an elevation of $24.4 \mathrm{~m}(80 \mathrm{ft})$. At the start of the simulation the pump was switched on. After $3 \mathrm{~min}$ simulation time, the pump was 
switched off and the resulting transient pressure waves were calculated for another 12 min simulation time.

\subsection{TAP Model Results}

TAP is a hydraulic modeling program that solves the fully dynamic onedimensional equations of continuity and momentum using a finite volume solution method. TAP simulates discontinuities in the HGL including open channel and pressure wave fronts using Roe's first order upwind scheme with an approximate Riemann solver (Roe 1981; Ridgway and Kumpula 2006, 2007; Ridgway 2008).

The channels or conduits in the system being analyzed are divided into uniformly spaced small cells. Time steps are automatically calculated to be stable and can vary throughout the model simulation. TAP employs a local time stepping scheme (Crossley 2003). For each cell and for each time step, the fully dynamic equations are solved, and cell averaged quantities of depth, area, velocity and flow rates are provided.

TAP easily models the geometry of the force main problem. In TAP the force main conduit was divided into $6.1 \mathrm{~m}(20 \mathrm{ft})$ segments for a total of 500 segments. The full conduit wave celerity for the TAP model was set to $121.9 \mathrm{~m} / \mathrm{s}(400 \mathrm{ft} / \mathrm{s})$. TAP utilizes a Preissmann slot above the conduit crown to track HGL when surcharged conditions occur (Preissmann 1961). The slot width in TAP is calculated from the user specified closed conduit wave speed (celerity). The Preissmann slot width, $B_{\text {slot }}$, is calculated as follows in TAP using the shallow water wave equation:

$$
B_{\text {slot }}=g \times A_{\text {full }} / a^{2}
$$

where:

$$
\begin{aligned}
g & =\text { gravitational acceleration } \\
A_{f u l l} & =\text { full conduit cross-sectional area, and } \\
a & =\text { closed conduit wave speed. }
\end{aligned}
$$

TAP has the ability to specify whether a junction is vented or not and both options were evaluated. With an unvented junction, less than atmospheric conditions can be calculated in the connected conduit cells. Figure 4.2 and Figure 4.3 show the TAP model results for the force main problem using both a vented and not vented junction at the discharge header. 


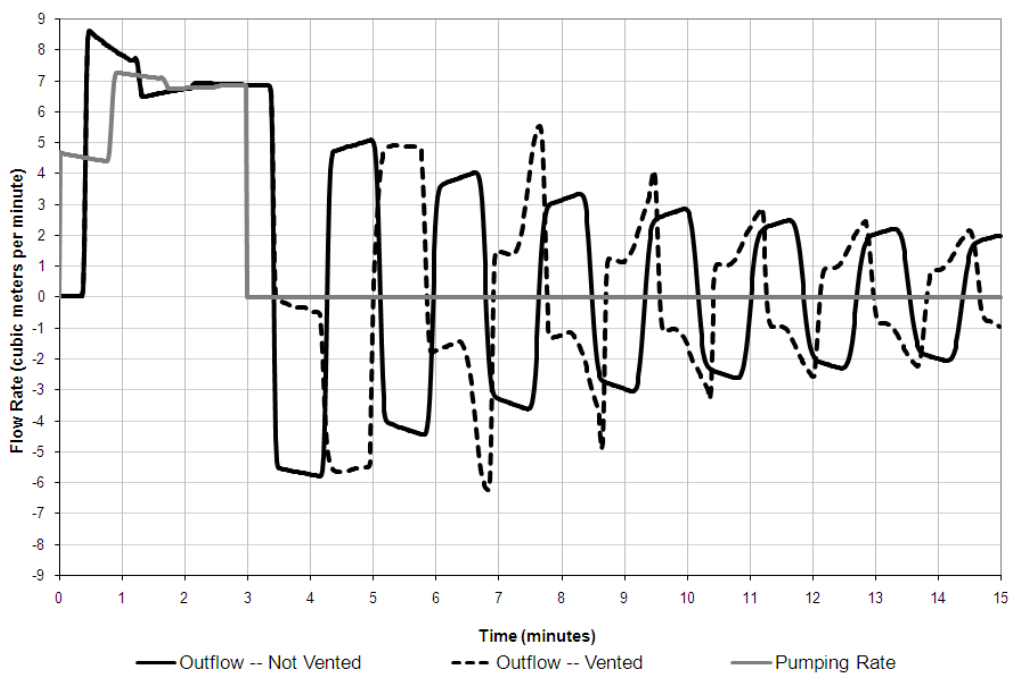

Figure 4.2 Downstream end flow rate and pumping rate versus time for vented and not vented tap model results.

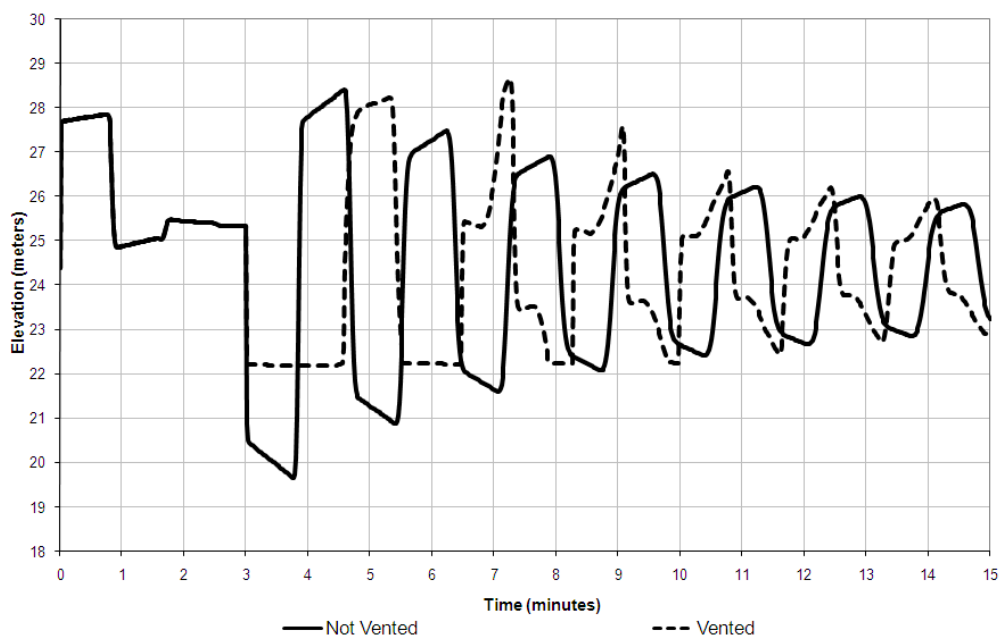

Figure 4.3 Discharge head versus time for vented and not vented TAP model results. 
The pressure waves that result from the pump turning on are the same whether the discharge header junction is specified to be vented or not. The pump turns on and initially the pumping rate is about $4.8 \mathrm{~m}^{3} / \mathrm{min}\left(2.8 \mathrm{ft}^{3} / \mathrm{s}\right)$. As expected, it takes the initial pressure wave about $25 \mathrm{~s}$ to reach the high reservoir $(10000 \mathrm{ft}$ at $400 \mathrm{ft} / \mathrm{s}=25 \mathrm{~s})$. The flow rate into the high reservoir is 0 for the first $25 \mathrm{~s}$ of the simulation. At about time $25 \mathrm{~s}$ (0.416 min), flow into the high reservoir is predicted to begin and the flow rates are predicted to be higher than the pumping rate for about the next $50 \mathrm{~s}(0.833 \mathrm{~min})$. The pumping rate and the flow rate into the reservoir become steadier and equal to about $6.8 \mathrm{~m}^{3} / \mathrm{min}$ $\left(4 \mathrm{ft}^{3} / \mathrm{s}\right)$ at about time $150 \mathrm{~s}(2.5 \mathrm{~min})$.

The pump is turned off at time $3 \mathrm{~min}$ and a large drop in pressure occurs in the force main at the discharge header. The resulting vented and unvented pressure oscillations have similar high side amplitudes but different shapes and periods of oscillation. The pressure in the discharge header drops to just below the force main crown elevation in the vented case. In the unvented case, the pressure drops significantly lower.

\subsection{WHAMO Model Results}

The WHAMO program is an accepted and widely used program for the analysis of closed conduit transients. WHAMO was used to validate the TAP model results for the not vented case. WHAMO solves the fully dynamic onedimensional equations of continuity and momentum using a matrix solution method. This method uses a Gaussian elimination technique with complete pivoting on the largest element. Junctions in WHAMO are always modeled as not vented.

The celerity in the WHAMO model was set to $121.9 \mathrm{~m} / \mathrm{s}(400 \mathrm{ft} / \mathrm{s})$ with a

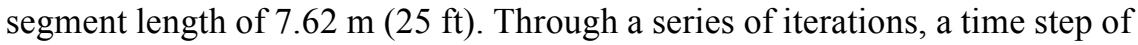
0.0015 was determined to yield the most reasonable and stable results. Figure 4.4 and Figure 4.5 show the WHAMO results as compared to the not vented TAP model results and the WHAMO model results agree closely with the TAP model results. As the oscillations continue, the WHAMO model results have more dispersion of the wave fronts than the TAP model results. This result is expected with the difference in solution technique. 


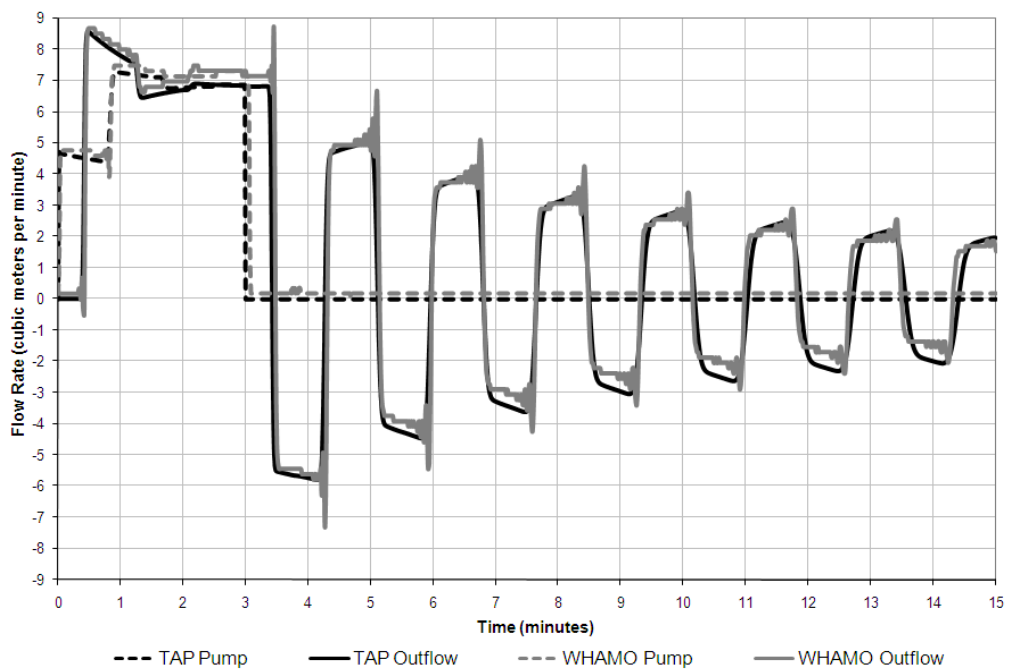

Figure 4.4 Downstream end flow rate and pumping rate versus time for WHAMO model results compared to not vented TAP model results.

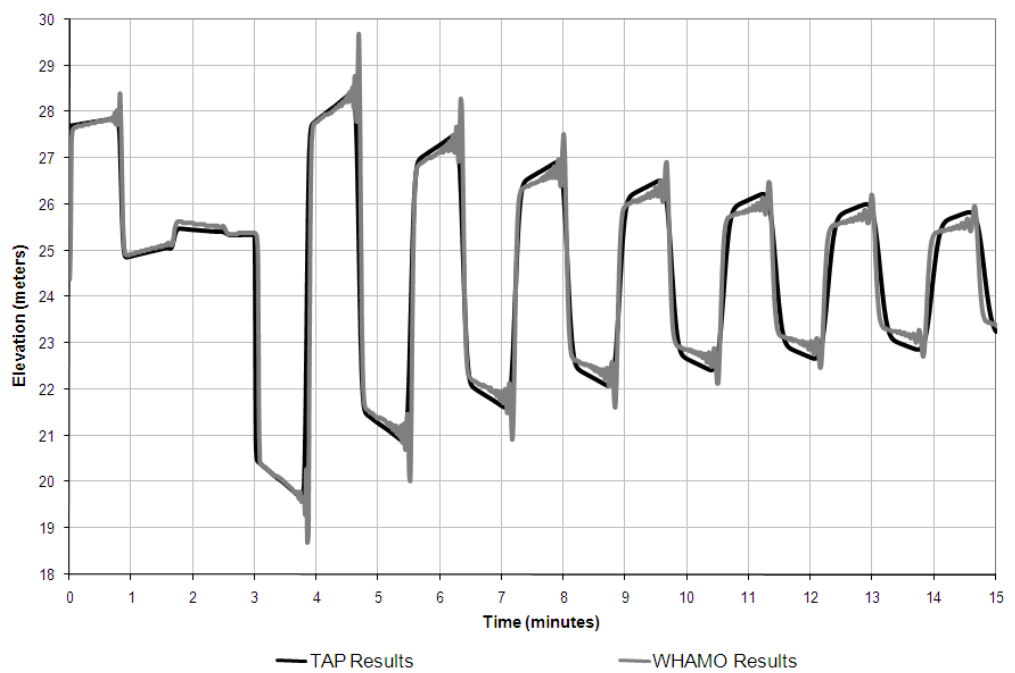

Figure 4.5 Discharge head versus time for WHAMO model results compared to not vented TAP model results. 


\subsection{SWMM5 Model Results}

SWMM5 was also able to accurately model the force main problem geometry with continuity errors $<3 \%$ for all simulations. Junctions in SWMM5 are always modeled as vented. The SWMM5 model was run using the dynamic wave solution technique. Initially, a single conduit (and the force main option) was used to model the force main with a $10 \mathrm{~s}$ time step. The force main pressure waves were very damped with this configuration. This is because the transient waves are numerically averaged across the single length of pipe during each time step. The continuity error for the run was $2.68 \%$.

The force main conduit was then broken up into ten segments, each $304.8 \mathrm{~m}$ ( $1000 \mathrm{ft}$ ) long, and the model was run with a range of computational time steps. A $4 \mathrm{~s}$ time step was determined to most accurately capture the transient waves. The SWMM5 results are shown in Figure 4.6 and Figure 4.7. The continuity error for the run was $2.94 \%$. The pressure wave results using the other time steps were more damped with similar continuity errors.

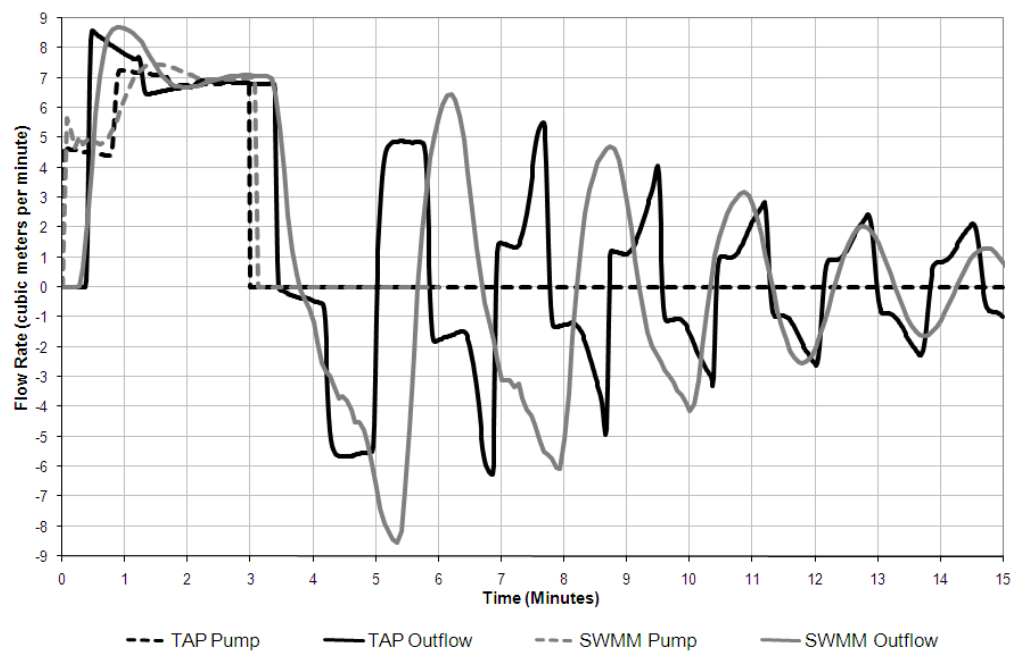

Figure 4.6 downstream end flow rate and pumping rate versus time for SWMM model results compared to vented TAP model results. 


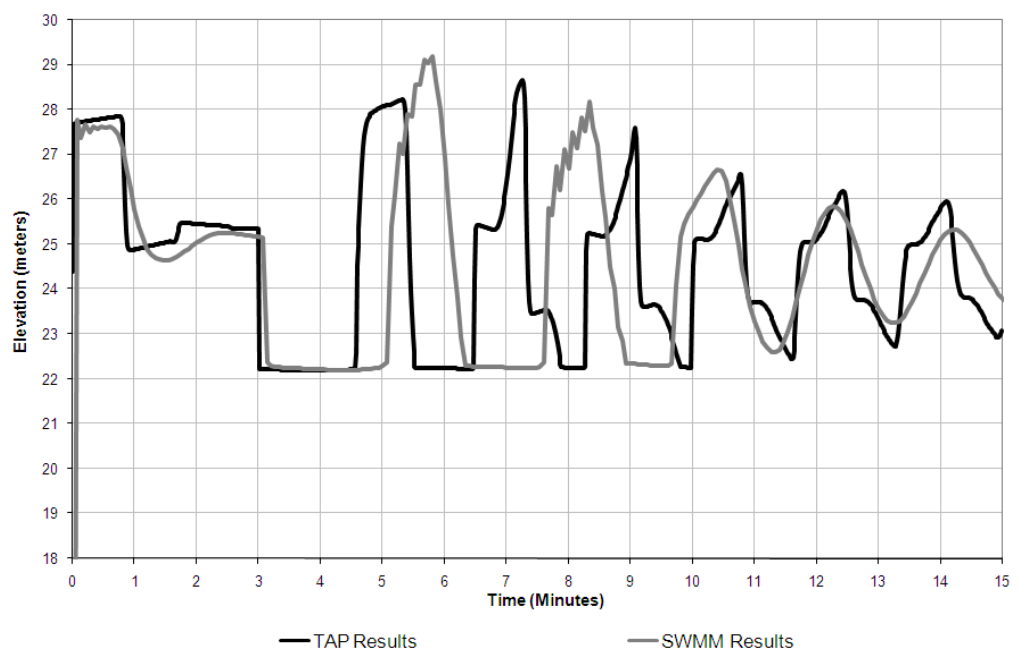

Figure 4.7 Discharge head versus time for SWMM model results compared to vented TAP model results.

\subsection{InfoWorks CS Model Results}

InfoWorks CS also utilizes a Preissmann slot above the conduit crown to track HGL when surcharged conditions occur. The slot width in InfoWorks CS is calculated from the user specified celerity ratio and conduit width parameters. These parameters, along with the size of the conduit, dictate the closed conduit wave speed being represented in the model.

The InfoWorks CS model was run using the both the pressure and the full solution technique methods. It was immediately evident that the default simulation parameters set in InfoWorks CS were causing the pressure waves to be highly damped with both of these solution methods.

The InfoWorks CS model parameters were adjusted using the full solution method to improve the results. The Preissmann slot width needed to provide a

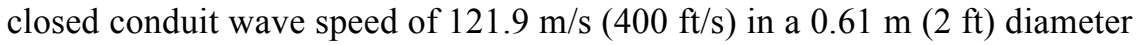
conduit is $0.000193 \mathrm{~m}(0.000632 \mathrm{ft})$ as calculated with Equation 4.1. In order to provide this Preissmann slot width, the celerity ratio was set to 80 , and the conduit width multiplier was set to 200 . The minimum time step of $1 \mathrm{~s}$ was used and the force main was divided into 25 segments, each $121.9 \mathrm{~m}(400 \mathrm{ft})$ long, by the InfoWorks CS program. Figure 4.8 and Figure 4.9 show the InfoWorks CS model results with the adjusted simulation parameters. 


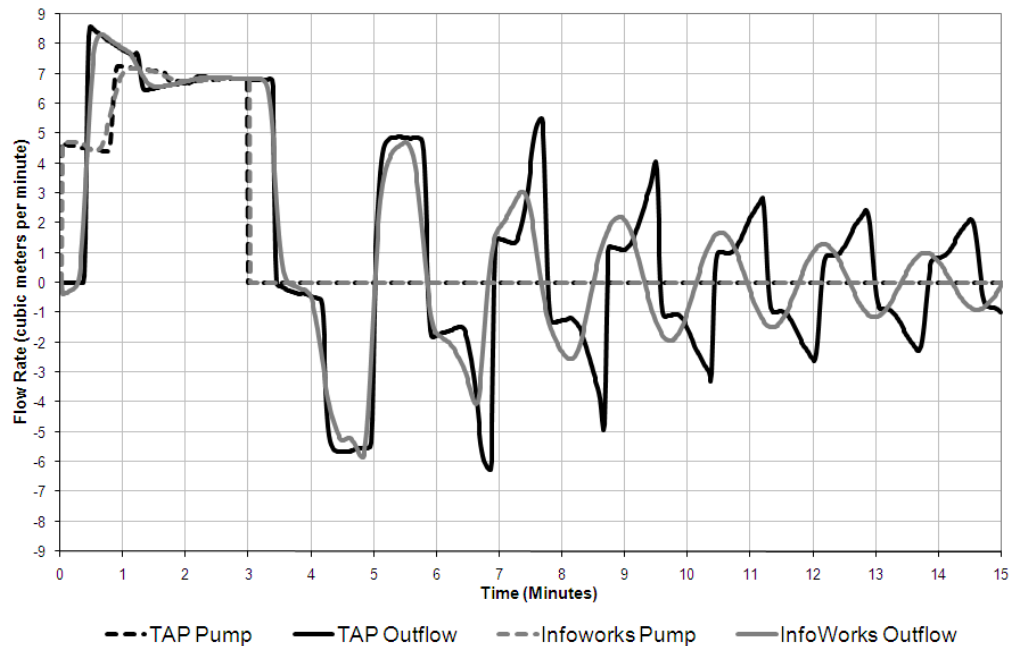

Figure 4.8 Downstream end flow rate and pumping rate versus time for InfoWorks CS model results compared to vented TAP model results.

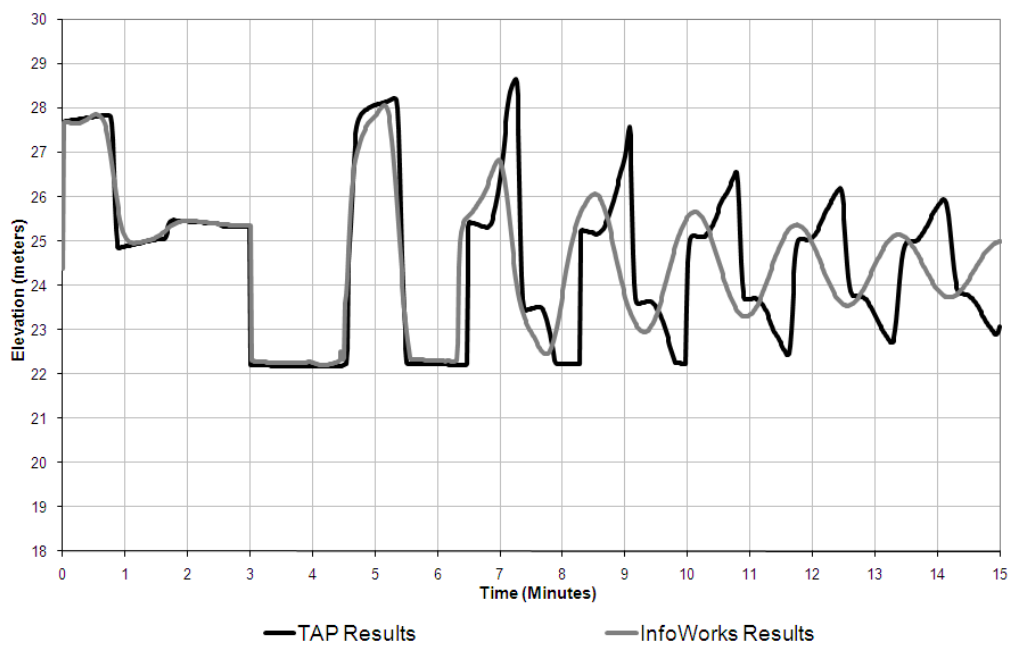

Figure 4.9 Discharge head versus time for InfoWorks CS model results compared to vented TAP model results. 


\subsection{Conclusions}

If set up carefully, SWMM5 and InfoWorks CS can closely simulate the resulting transient pressure waves that occur in force mains when pumps turn on and off. This conclusion applies only if the force main is actually vented at high points such as the pump station discharge header.

Force main calculations with SWMM5 are more realistic with multiple shorter (but stable length) conduit segments. The SWMM5 force main routine allows only one long force main conduit and having one long force main segment yields over damped results.

SWMM5 and InfoWorks CS typically over damp the resulting pressure waves and oscillations in force mains when pumps turn on and off. This conclusion is true when force mains are set up in larger wastewater collection system models with typical time steps and model parameters.

Force mains (and conduits) are always vented in SWMM5 and InfoWorks CS but not in reality. Therefore SWMM5 and InfoWorks CS cannot model less than atmospheric conditions in force mains and conduits. These programs are not recommended to be used to predict the formation and collapse of vapor cavities in force mains and to design air entrance and release valves.

SWMM5 and InfoWorks CS are good tools to track pumping volumes and peak rates through wastewater collection systems.

TAP and WHAMO simulate force main transients correctly and are proper tools for designing pump stations (surge control and air or vacuum valves). TAP and WHAMO do not underestimate and damp the resulting pressure waves and oscillations when pumps turn on and off. TAP and WHAMO can model less than atmospheric conditions in force mains and conduits. TAP has the advantage of allowing model junctions to be specified as vented or not vented.

\section{References}

Crossley, A. J. (2003). "Local time stepping for modeling open channel flows." J. Hydr. Engrg., 129(6), 455-462.

Preissmann, A. (1961). "Propagation des intumescences dans les canaux et rivières", 1st Congress of the French Association for Computation (AFCALTI), September, Grenoble.

Ridgway, K. 2008. "Evaluating Surge Potential in CSO Tunnels." Journal of Water Management Modeling R229-14. doi: 10.14796/JWMM.R229-14. 
Ridgway, K. and G.J. Kumpula. 2006. "Surge Modeling in Sewers using the Transient Analysis Program (TAP)." Journal of Water Management Modeling R227-06. doi: 10.14796/JWMM.R227-06.

Ridgway, K. and G.J. Kumpula. 2007. "Surge Modeling in Sewers using Alternative Hydraulic Software Programs." Journal of Water Management Modeling R228-10. doi: 10.14796/JWMM.R228-10.

Roe, P. L. (1981). "Approximate Riemann solvers, parameter vectors, and difference schemes.” J. Comp. Physics, 43, 357-372. 